\title{
ON THE CORRELATION STRUCTURE OF A LÉVY-DRIVEN QUEUE
}

\author{
ABDELGHAFOUR ES-SAGHOUANI,* University of Amsterdam \\ MICHEL MANDJES, ${ }^{* *}$ University of Amsterdam, CWI, and EURANDOM
}

\begin{abstract}
In this paper we consider a single-server queue with Lévy input and, in particular, its workload process $\left(Q_{t}\right)_{t \geq 0}$, with a focus on the correlation structure. With the correlation function defined as $r(t):=\operatorname{cov}\left(Q_{0}, Q_{t}\right) / \operatorname{var}\left(Q_{0}\right)$ (assuming that the workload process is in stationarity at time 0 ), we first determine its transform $\int_{0}^{\infty} r(t) \mathrm{e}^{-\vartheta t} \mathrm{~d} t$. This expression allows us to prove that $r(\cdot)$ is positive, decreasing, and convex, relying on the machinery of completely monotone functions. We also show that $r(\cdot)$ can be represented as the complementary distribution function of a specific random variable. These results are used to compute the asymptotics of $r(t)$, for large $t$, for the cases of light-tailed and heavy-tailed Lévy inputs.
\end{abstract}

Keywords: Lévy process; reflection; correlation; complete monotonicity

2000 Mathematics Subject Classification: Primary 60K25; 60G51; 90B05

\section{Introduction}

Consider a queueing system and, more particularly, its workload process $\left(Q_{t}\right)_{t \geq 0}$. Where one usually focuses on the characterization of the (transient or steady-state) workload, another interesting problem relates to the identification of the correlation function

$$
r(t):=\frac{\operatorname{cov}\left(Q_{0}, Q_{t}\right)}{\operatorname{var}\left(Q_{0}\right)}
$$

For several queueing systems, this correlation function has been explicitly computed; Morse [21], for instance, analyzed the number of customers in the M/M/1 queue. Often explicit formulae were hard to obtain, but the analysis simplified greatly when looking at the transform

$$
\rho(\vartheta):=\int_{0}^{\infty} r(t) \mathrm{e}^{-\vartheta t} \mathrm{~d} t .
$$

Beneš [6] managed to compute $\rho(\cdot)$ for the workload in the M/G/1 queue; relying on the concept of complete monotonicity, Ott [22] elegantly proved that, in this case, $r(\cdot)$ is positive, decreasing, and convex. We further mention the survey by Reynolds [23] and interesting results by Abate and Whitt [2].

The primary aim of this paper is to extend the results mentioned above to the class of singleserver queues fed by Lévy processes. Note that the M/G/1 queue is contained in this class (then

Received 28 November 2007; revision received 27 October 2008.

* Postal address: Korteweg-de Vries Institute for Mathematics, University of Amsterdam, Plantage Muidergracht 24,

1018 TV Amsterdam, The Netherlands. Email address: aessagho@science.uva.nl

** Postal address: CWI, PO Box 94079, 1090 GB Amsterdam, The Netherlands.

Email address: mmandjes@ science.uva.nl 
the Lévy process under consideration is a compound Poisson process with drift). We could expect that such an extension is possible, as the classical Pollaczek-Khinchine result for the M/G/1 queue carries over to queues with general Lévy input; see [25] for an early reference and, for an extensive account of fluctuation theory for Lévy processes, see [9] and the references therein, and [17]. The only condition we usually need to impose in order to obtain explicit results is that no negative jumps are allowed.

In more detail, the setting we consider is the following. We define a 'net input process' $\left(X_{t}\right)_{t \geq 0}$, which is assumed to be a Lévy process with no negative jumps. Then the workload process $\left(Q_{t}\right)_{t \geq 0}$ is defined as the reflected process of $\left(X_{t}\right)_{t \geq 0}$ at 0 . Because of the lack of explicit formulae for the probability distributions of the processes considered, we will work most of the time with their Laplace transforms; in our analysis, the Laplace exponent $\varphi(\cdot)$ of the process $\left(X_{t}\right)_{t \geq 0}$, as well as its inverse $\psi(\cdot)$, plays an important role.

We first obtain an explicit expression, in terms of $\varphi(\cdot)$ and $\psi(\cdot)$, of the transform $\rho(\cdot)$ of the correlation function. Using the concept of complete monotonicity, we use this transform to establish a series of structural properties of the correlation function, viz. we prove that $r(\cdot)$ is positive, decreasing, and convex. These results indeed generalize those obtained in [2] and [22] for the M/G/1 queue. We then consider the asymptotic behavior of $r(t)$ for large $t$. For light-tailed Lévy input, these asymptotics are essentially exponential; for the M/G/1 case, they resemble those of the busy period. For heavy-tailed input, we can use results for regularly varying functions, e.g. Karamata's Tauberian theorem, to obtain the asymptotics of $r(\cdot)$.

This paper is organized as follows. In Section 2 we obtain the Laplace transform of the correlation function and in Section 3 we study its structural properties. The cases of light-tailed and heavy-tailed inputs are treated in Sections 4 and 5, respectively. Concluding remarks are found in Section 6.

\section{Laplace transform of the correlation function}

In this section we find an expression for the transform $\rho(\cdot)$ of the correlation function. We start this section, however, with a formal introduction of our queueing system.

\subsection{Lévy processes}

Let $\left(X_{t}\right)_{t \geq 0}$ be a Lévy process without negative jumps, with drift $\mathrm{E}\left(X_{1}\right)<0$. Its Laplace exponent is given by the function $\varphi(\cdot):[0, \infty) \mapsto[0, \infty)$, i.e. $\varphi(\alpha):=\log \mathrm{E}\left(\exp \left[-\alpha X_{1}\right]\right)$. It is known that $\varphi(\cdot)$ is increasing and convex on $[0, \infty)$ with slope $\varphi^{\prime}(0)=-\mathrm{E}\left(X_{1}\right)$ at the origin. Therefore, the inverse $\psi(\cdot)$ of $\varphi(\cdot)$ is well defined on $[0, \infty)$. In the sequel we also require that $X_{t}$ is not a subordinator, i.e. a monotone process; thus, $X_{1}$ has probability mass on the positive half-line, which implies that $\lim _{\alpha \rightarrow-\infty} \varphi(\alpha)=\infty$.

Important examples of such Lévy processes are the following.

(i) Brownian motion with drift. We write $X \in \operatorname{Bm}\left(\mu, \sigma^{2}\right)$ when $\varphi(\alpha)=-\alpha \mu+\frac{1}{2} \alpha^{2} \sigma^{2}$.

(ii) Compound Poisson with drift. Jobs arrive according to a Poisson process of rate $\lambda$; the jobs $B_{1}, B_{2}, \ldots$ are independent and identically distributed samples from a distribution with Laplace transform $\beta(\alpha):=\mathrm{E}\left(\mathrm{e}^{-\alpha B}\right)$; the storage system is continuously depleted at a rate -1 . We write $X \in \mathrm{CP}(\lambda, \beta(\cdot))$; it can be verified that $\varphi(\alpha)=\alpha-\lambda+\lambda \beta(\alpha)$.

\subsection{Reflected Lévy processes; queues}

We consider the reflection of $\left(X_{t}\right)_{t \geq 0}$ at 0 , which we denote by $\left(Q_{t}\right)_{t \geq 0}$. It is formally introduced as follows; see, for instance, [4, Chapter IX]. Define the increasing process $\left(L_{t}\right)_{t \geq 0}$ 
by

$$
L_{t}=-\inf _{0 \leq s \leq t} X_{s}
$$

Then the reflected process (or workload process or queueing process) $\left(Q_{t}\right)_{t \geq 0}$ is given through

$$
Q_{t}:=X_{t}+\max \left\{L_{t}, Q_{0}\right\}
$$

observe that $Q_{t} \geq 0$ for all $t \geq 0$. Then the steady-state distribution of $Q:=\lim _{t \rightarrow \infty} Q_{t}$ is characterized in [25]:

$$
\kappa(\alpha):=\mathrm{E}\left(\mathrm{e}^{-\alpha Q}\right)=\frac{\alpha \varphi^{\prime}(0)}{\varphi(\alpha)} ;
$$

for the special case of CP input, this is the celebrated Pollaczek-Khinchine formula. This relation reveals all moments of the steady-state queue $Q$ and, in particular, its mean and variance:

$$
\mu:=\mathrm{E}(Q)=-\left.\frac{\mathrm{d}}{\mathrm{d} \alpha} \frac{\alpha \varphi^{\prime}(0)}{\varphi(\alpha)}\right|_{\alpha \downarrow 0}=\frac{\varphi^{\prime \prime}(0)}{2 \varphi^{\prime}(0)}
$$

and, similarly,

$$
v:=\operatorname{var}(Q)=\frac{1}{4}\left(\frac{\varphi^{\prime \prime}(0)}{\varphi^{\prime}(0)}\right)^{2}-\frac{1}{3} \frac{\varphi^{\prime \prime \prime}(0)}{\varphi^{\prime}(0)},
$$

which from now on are assumed to be finite.

\subsection{Correlation structure of the queue}

In this paper we are interested in the correlation structure of the queue process $\left(Q_{t}\right)_{t \geq 0}$. Our analysis relies on the following useful relation (see, e.g. [4, Section IX.3] and [16]):

$$
\mathrm{E}\left(\exp \left[-\alpha Q_{T}\right] \mid Q_{0}=q\right)=\frac{\vartheta}{\vartheta-\varphi(\alpha)}\left(\mathrm{e}^{-\alpha q}-\alpha \frac{\mathrm{e}^{-\psi(\vartheta) q}}{\psi(\vartheta)}\right),
$$

where $T$ is exponentially distributed with mean $\vartheta^{-1}$, independently of the Lévy process. (As an aside, we mention that (2.4) implies the Pollaczek-Khinchine formula in at least two ways: (a) let $\vartheta \downarrow 0$, so that $T$ corresponds with some epoch infinitely far away, and use elementary calculus (l'Hôpital's rule); (b) find $\mathrm{E}\left(\exp \left[-\alpha Q_{T}\right]\right)$ by deconditioning, use that in stationarity, $\mathrm{E}\left(\exp \left[-\alpha Q_{T}\right]\right)$ should coincide with $\mathrm{E}\left(\exp \left[-\alpha Q_{0}\right]\right)$, and then solve $\mathrm{E}\left(\exp \left[-\alpha Q_{0}\right]\right)$ )

Equation (2.4) enables us to find explicitly the Laplace transform $\rho(\cdot)$ of

$$
r(t):=\operatorname{corr}\left(Q_{0}, Q_{t}\right)=\frac{\operatorname{cov}\left(Q_{0}, Q_{t}\right)}{\sqrt{\operatorname{var}\left(Q_{0}\right) \operatorname{var}\left(Q_{t}\right)}}=\frac{\mathrm{E}\left(Q_{0} Q_{t}\right)-\left(\mathrm{E}\left(Q_{0}\right)\right)^{2}}{\operatorname{var}\left(Q_{0}\right)},
$$

as we show now. Here it is assumed that the system is in steady state at time 0 , that is, $Q_{0}$ obeys the 'generalized Pollaczek-Khinchine' formula, (2.1). First realize that

$$
\mathrm{E}\left(\exp \left[-\alpha Q_{T}\right] \mid Q_{0}=q\right)=\int_{0}^{\infty} \vartheta \mathrm{e}^{-\vartheta t} \mathrm{E}\left(\exp \left[-\alpha Q_{t}\right] \mid Q_{0}=q\right) \mathrm{d} t
$$

By differentiation with respect to $\alpha$ and subsequently letting $\alpha \downarrow 0$, we obtain

$$
\int_{0}^{\infty} \vartheta \mathrm{e}^{-\vartheta t} \mathrm{E}\left(Q_{t} \mid Q_{0}=q\right) \mathrm{d} t=-\frac{\varphi^{\prime}(0)}{\vartheta}+q+\frac{\mathrm{e}^{-\psi(\vartheta) q}}{\psi(\vartheta)} .
$$


Concentrate on the Laplace transform $\gamma(\vartheta)$ of $\operatorname{cov}\left(Q_{0}, Q_{t}\right)$. Straightforward calculus reveals that

$$
\begin{aligned}
\gamma(\vartheta) & :=\int_{0}^{\infty} \operatorname{cov}\left(Q_{0}, Q_{t}\right) \mathrm{e}^{-\vartheta t} \mathrm{~d} t \\
& =\int_{0}^{\infty}\left(\mathrm{E}\left(Q_{0} Q_{t}\right)-\mu^{2}\right) \mathrm{e}^{-\vartheta t} \mathrm{~d} t \\
& =\int_{0}^{\infty} \int_{0}^{\infty} q \mathrm{E}\left(Q_{t} \mid Q_{0}=q\right) \mathrm{e}^{-\vartheta t} \mathrm{dP}\left(Q_{0} \leq q\right) \mathrm{d} t-\frac{\mu^{2}}{\vartheta}
\end{aligned}
$$

it is assumed that the queue is in stationarity at time 0 (and, hence, it is in stationarity at time $t$ as well). By invoking (2.5) we find that the expression in the previous display equals

$$
\begin{aligned}
\int_{0}^{\infty} & \frac{q}{\vartheta}\left(-\frac{\varphi^{\prime}(0)}{\vartheta}+q+\frac{\mathrm{e}^{-\psi(\vartheta) q}}{\psi(\vartheta)}\right) \mathrm{dP}\left(Q_{0} \leq q\right)-\frac{\mu^{2}}{\vartheta} \\
= & -\frac{\mu \varphi^{\prime}(0)}{\vartheta^{2}}+\frac{v}{\vartheta}+\frac{1}{\vartheta \psi(\vartheta)} \mathrm{E}\left(Q_{0} \exp \left[-\psi(\vartheta) Q_{0}\right]\right) .
\end{aligned}
$$

From the generalized Pollaczek-Khinchine formula, (2.1), we obtain, by differentiating,

$$
\mathrm{E}\left(Q_{0} \exp \left[-\alpha Q_{0}\right]\right)=\varphi^{\prime}(0)\left(-\frac{1}{\varphi(\alpha)}+\alpha \frac{\varphi^{\prime}(\alpha)}{(\varphi(\alpha))^{2}}\right) .
$$

Inserting this relation, in addition to (2.2), into (2.6) we obtain the Laplace transform of $\operatorname{cov}\left(Q_{0}, Q_{t}\right)$ :

$$
\gamma(\vartheta)=-\frac{\varphi^{\prime \prime}(0)}{2 \vartheta^{2}}+\frac{v}{\vartheta}+\frac{\varphi^{\prime}(0)}{\vartheta^{2}}\left(\frac{1}{\vartheta \psi^{\prime}(\vartheta)}-\frac{1}{\psi(\vartheta)}\right)
$$

This also trivially provides us with the Laplace transform of $\operatorname{corr}\left(Q_{0}, Q_{t}\right)$, as stated in the following theorem. When specializing to CP input, we retrieve Equation (6.2) of [6].

Theorem 2.1. For any $\vartheta \geq 0$, and $v$ as in (2.3),

$$
\rho(\vartheta):=\int_{0}^{\infty} r(t) \mathrm{e}^{-\vartheta t} \mathrm{~d} t=\frac{\gamma(\vartheta)}{v}=\frac{1}{\vartheta}-\frac{\varphi^{\prime \prime}(0)}{2 v \vartheta^{2}}+\frac{\varphi^{\prime}(0)}{v \vartheta^{2}}\left(\frac{1}{\vartheta \psi^{\prime}(\vartheta)}-\frac{1}{\psi(\vartheta)}\right) .
$$

Remark 2.1. Using the generalized Pollaczek-Khinchine formula, (2.1), it is readily verified that the result in Theorem 2.1 can be simplified to

$$
\rho(\vartheta)=\frac{1}{\vartheta}-\frac{1}{v}\left(\frac{\varphi^{\prime \prime}(0)}{2 \vartheta^{2}}+\frac{\kappa^{\prime}(\psi(\vartheta))}{\vartheta \psi(\vartheta)}\right)
$$

Example 2.1. Consider the situation in which $\left(X_{t}\right)_{t \geq 0}$ corresponds to standard Brownian motion decreased by a linear drift (say of rate 1 , so $X \in \operatorname{Bm}(-1,1)$ ). In other words, the Laplace exponent of the Lévy process is given by $\varphi(\alpha)=\alpha+\frac{1}{2} \alpha^{2}$ and its inverse is $\psi(\vartheta)=-1+\sqrt{1+2 \vartheta}$. Now consider the workload process $\left(Q_{t}\right)_{t \geq 0}$ and its correlation function. The above theory shows that the Laplace transform of $r(\cdot)$ is given by

$$
\rho(\vartheta)=\frac{1}{\vartheta}-\frac{2}{\vartheta^{2}}+\frac{2}{\vartheta^{3}}(\sqrt{1+2 \vartheta}-1) .
$$

It turns out to be possible to explicitly invert $\rho(\cdot)$ :

$$
r(t)=2\left(1-2 t-t^{2}\right)\left(1-\Phi_{\mathrm{N}}(\sqrt{t})\right)+2 \sqrt{t}(1+t) \phi_{\mathrm{N}}(\sqrt{t}),
$$

where $\Phi_{\mathrm{N}}(\cdot)$ and $\phi_{\mathrm{N}}(\cdot)$ are the standard normal distribution and the standard normal density, respectively. Equation (2.8) is in agreement with the results in [1] and [18, Section 12.1]. 


\section{Structural properties of the correlation function}

In this section we concentrate on the derivation of a number of key structural properties of the correlation function $r(\cdot)$. More specifically, relying on the concept of completely monotone functions [7], [22], we prove in Theorem 3.1, below, that $r(\cdot)$ is a positive, decreasing, and convex function. To this end, we first establish a number of auxiliary results; a key result is the following.

Proposition 3.1. Define

$$
\xi(\vartheta):=\frac{1}{\mu}\left(\frac{1}{\vartheta \psi^{\prime}(\vartheta)}-\frac{1}{\psi(\vartheta)}\right) .
$$

Then $\xi(\vartheta)$ is the Laplace transform of a (nonnegative) random variable $Z$.

Remark 3.1. The Laplace transform of the stationary-excess distribution $Z_{\mathrm{e}}$ associated with $Z$ is given by (see [2])

$$
\xi_{\mathrm{e}}(\vartheta)=\frac{\xi(\vartheta)-1}{\vartheta \xi^{\prime}(0)}=\frac{\varphi^{\prime \prime}(0)}{2 v \vartheta}(1-\xi(\vartheta)) .
$$

Hence, the first moment of $Z$ is $2 v / \varphi^{\prime \prime}(0)$.

To prove Proposition 3.1, we need a number of lemmas. These are stated and proved now. They extensively use the concept of complete monotonicity [7], [14, pp. 439-442]. The class $\mathcal{C}$ of completely monotone functions is defined in Appendix A, where a series of standard properties is also given.

Lemma 3.1. We have $\psi^{\prime}(\vartheta) \in \mathcal{C}$.

Proof. Consider, for $x \geq 0$,

$$
T_{x}:=\inf \left\{t \geq 0: X_{t}=-x\right\} .
$$

Then $T_{x}$ is a Lévy process with Laplace exponent $-\psi(\vartheta)$; see, e.g. [24, Theorem 46.3]. More specifically, $T_{x}$ is a subordinator. Now apply Lemma A.2.

Lemma 3.2. If $f(\alpha) \in \mathcal{C}$ then so does

$$
\frac{f(0)-f(\alpha)+\alpha f^{\prime}(\alpha)}{\alpha^{2}} .
$$

Proof. This is a consequence of subsequently applying Lemma A.1(iv) and (v).

Lemma 3.3. For $\sigma^{2}>0$ and measure $\Pi_{\varphi}(\cdot)$ such that $\int_{(0, \infty)} \min \left\{1, x^{2}\right\} \Pi_{\varphi}(\mathrm{d} x)<\infty$,

$$
\frac{\alpha \varphi^{\prime}(\alpha)-\varphi(\alpha)}{\alpha^{2}}=\frac{1}{2} \sigma^{2}+\frac{1}{\alpha^{2}} \int_{(0, \infty)}\left(1-\mathrm{e}^{-\alpha x}-\alpha x \mathrm{e}^{-\alpha x}\right) \Pi_{\varphi}(\mathrm{d} x) \in \mathcal{C} .
$$

Proof. The Laplace exponent $\varphi(\alpha)$ can be written as, with $\sigma^{2}>0$ and measure $\Pi_{\varphi}(\cdot)$ such that $\int_{(0, \infty)} \min \left\{1, x^{2}\right\} \Pi_{\varphi}(\mathrm{d} x)<\infty$,

$$
\varphi(\alpha)=-\alpha \mu+\frac{1}{2} \alpha^{2} \sigma^{2}+\int_{(0, \infty)}\left(\mathrm{e}^{-\alpha x}-1+\alpha x \mathbf{1}_{(0,1)}\right) \Pi_{\varphi}(\mathrm{d} x),
$$

which immediately yields the equality in (3.3). The claim that this function is in $\mathcal{C}$ follows from the fact that any positive constant is in $\mathcal{C}$, Lemma 3.2, and Lemma A.1(i). 
Proof of Proposition 3.1. We first decompose

$$
\frac{1}{\vartheta \psi^{\prime}(\vartheta)}-\frac{1}{\psi(\vartheta)}=\eta_{1}(\vartheta) \eta_{2}(\vartheta)
$$

where

$$
\eta_{1}(\vartheta):=\frac{\psi(\vartheta)}{\vartheta} \quad \text { and } \quad \eta_{2}(\vartheta):=\frac{1}{\psi(\vartheta) \psi^{\prime}(\vartheta)}-\frac{\vartheta}{(\psi(\vartheta))^{2}} .
$$

Because of the (generalized version of the) Pollaczek-Khinchine formula, (2.1), we have $\alpha / \varphi(\alpha) \in \mathcal{C}$; now applying Lemma A.1(iii), in conjunction with Lemma 3.1, we obtain $\eta_{1}(\vartheta) \in \mathcal{C}$.

To prove that $\eta_{2}(\vartheta) \in \mathcal{C}$ also, we first recall from Lemma 3.3 that $\left(\alpha \varphi^{\prime}(\alpha)-\varphi(\alpha)\right) / \alpha^{2} \in \mathcal{C}$. Again, applying Lemma A.1(iii), in conjunction with Lemma 3.1, it follows that $\eta_{2}(\vartheta) \in \mathcal{C}$.

As both $\eta_{1}(\vartheta)$ and $\eta_{2}(\vartheta)$ are in $\mathcal{C}$, Lemma A.1(ii) yields $\xi(\vartheta) \in \mathcal{C}$. Applying l'Hôpital's rule twice, and using the fact that $\psi^{\prime \prime}(0)\left(\varphi^{\prime}(0)\right)^{3}=-\varphi^{\prime \prime}(0)$, it is readily verified that

$$
\xi(0)=\lim _{\vartheta \downarrow 0} \xi(\vartheta)=1 .
$$

Now Theorem A.1 yields the result.

Let $\rho^{(1)}(\vartheta)$ and $\rho^{(2)}(\vartheta)$ be the Laplace transforms of $r^{\prime}(t):=(\mathrm{d} / \mathrm{d} t) r(t)$ and $r^{\prime \prime}(t):=$ $\left(\mathrm{d}^{2} / \mathrm{d} t^{2}\right) r(t)$. Their expressions are given respectively as follows:

$$
\begin{aligned}
& \rho^{(1)}(\vartheta):=\int_{0}^{\infty} r^{\prime}(t) \mathrm{e}^{-\vartheta t} \mathrm{~d} t=-\frac{\varphi^{\prime \prime}(0)}{2 v \vartheta}(1-\xi(\vartheta))=-\xi_{\mathrm{e}}(\vartheta), \\
& \rho^{(2)}(\vartheta):=\int_{0}^{\infty} r^{\prime \prime}(t) \mathrm{e}^{-\vartheta t} \mathrm{~d} t=\frac{\varphi^{\prime \prime}(0)}{2 v} \xi(\vartheta),
\end{aligned}
$$

for $\vartheta \geq 0$. Here the properties that $r(0)=1$ and

$$
r^{\prime}(0)=\lim _{\varepsilon \downarrow 0} \frac{\mathrm{E}\left(Q_{0} Q_{\varepsilon}\right)-\left(\mathrm{E}\left(Q_{0}\right)\right)^{2}}{\varepsilon \operatorname{var}\left(Q_{0}\right)}=\lim _{\varepsilon \downarrow 0} \frac{\mathrm{E}\left(Q_{0} X_{\varepsilon}\right)}{\varepsilon \operatorname{var}\left(Q_{0}\right)}=-\frac{\varphi^{\prime \prime}(0)}{2 v},
$$

in conjunction with integration by parts, have been used.

Theorem 3.1. The correlation function $r(t)$ is positive, decreasing, and convex. Furthermore, $r(t)$ can be written as the tail of the stationary-excess distribution function associated with $Z$, i.e. $r(t)=\mathrm{P}\left(Z_{\mathrm{e}}>t\right)$. If $Z$ has a finite second moment then $r(t)$ is integrable and

$$
\int_{0}^{\infty} r(t) \mathrm{d} t=\frac{1}{8 v} \frac{\varphi^{(4)}(0)}{\varphi^{\prime}(0)^{2}}-\frac{5}{12 v} \frac{\varphi^{\prime \prime}(0) \varphi^{(3)}(0)}{\varphi^{\prime}(0)^{3}}+\frac{1}{4 v} \frac{\varphi^{\prime \prime}(0)^{3}}{\varphi^{\prime}(0)^{4}} .
$$

Proof. Convexity follows from the expression for $\rho^{(2)}(\vartheta)$ in (3.5); it is concluded from Proposition 3.1 that $\rho^{(2)}(\vartheta) \in \mathcal{C}$; thus, $r^{\prime \prime}(t)$ is nonnegative (for $t \geq 0$ ). The monotonicity follows from the expression for $\rho^{(1)}(\vartheta)$ in (3.4), by applying Lemma A.1(iv) to $\rho^{(2)}(\vartheta) \in \mathcal{C}$; we find that $-\rho^{(1)}(\vartheta)$ is in $\mathcal{C}$, implying that $r^{\prime}(t) \leq 0$ (for $t \geq 0$ ). Then it is easily verified that applying Lemma A.1(iv) to $-\rho^{(1)}(\vartheta) \in \mathcal{C}$, in conjunction with (2.7), implies that $\rho(\vartheta) \in \mathcal{C}$, and, hence, $r(t) \geq 0$ (for $t \geq 0$ ).

Observe that combining (2.7) and (3.2) yields

$$
\rho(\vartheta)=\frac{1-\xi_{\mathrm{e}}(\vartheta)}{\vartheta}
$$


It is straightforward to verify that the right-hand side of the previous display is just the Laplace transform of $\mathrm{P}\left(Z_{\mathrm{e}}>t\right)$. It is concluded that $r(t)=\mathrm{P}\left(Z_{\mathrm{e}}>t\right)$ by the uniqueness of the Laplace transform. Equation (3.6) follows, after considerable calculus (i.e. application of l'Hôpital's rule several times and various series expansions), by evaluating

$$
\int_{0}^{\infty} r(t) \mathrm{d} t=\rho(0)=\lim _{\vartheta \downarrow 0} \rho(\vartheta) ;
$$

it is noted that $\varphi^{(4)}(0)$ exists if the second moment of $Z$ is finite.

\section{Correlation asymptotics for light-tailed input}

When $\varphi(\cdot)$ has an analytic continuation for $\alpha<0$, we are in the regime of light tails; as $a$ fortiori then all moments $(-1)^{n} \varphi^{(n)}(0)$ of $X_{1}$ exist. When $\left(X_{t}\right)_{t>0}$ does not correspond to a decreasing subordinator, we also have $\lim _{\alpha \rightarrow-\infty} \varphi(\alpha)=\infty$. Bearing in mind the facts that $\varphi(\cdot)$ has a positive slope at the origin and that convexity of $\varphi(\cdot)$ implies continuity, there is a unique minimizer $\zeta<0$ such that $\varphi(\zeta)<0, \varphi^{\prime}(\zeta)=0$, and $\varphi^{\prime \prime}(\zeta)>0$.

In this situation, $\psi(\cdot)$ is also well defined for negative arguments; more precisely, for all $\vartheta \geq \varphi(\zeta)$, the inverse $\psi(\vartheta)$ has a meaningful interpretation. In fact, $\vartheta^{\star}:=\varphi(\zeta)$ can be regarded as a branching point. We thus see that Theorem 2.1 does not only apply for $\vartheta \geq 0$, but also for $\vartheta \in\left[\vartheta^{\star}, 0\right)$. Around $\zeta$, we can write $\varphi(\cdot)$ as

$$
\varphi(\alpha)=\varphi(\zeta)+\frac{1}{2}(\alpha-\zeta)^{2} \varphi^{\prime \prime}(\zeta)+O\left((\alpha-\zeta)^{3}\right)
$$

and, hence, for $\theta \downarrow \vartheta^{\star}$,

$$
\psi(\vartheta)-\zeta \sim \sqrt{\frac{2}{\varphi^{\prime \prime}(\zeta)}} \sqrt{\vartheta-\varphi(\zeta)}=\sqrt{\frac{2}{\varphi^{\prime \prime}(\zeta)}} \sqrt{\vartheta-\vartheta^{\star}}
$$

(where ' $\sim$ ' indicates that the ratio of the left- and right-hand sides tends to 1). Routine calculations reveal that, for $\theta \downarrow \vartheta^{\star}, \rho(\vartheta)$ looks like

$$
\frac{1}{v}\left(-\frac{\varphi^{\prime \prime}(0)}{2\left(\vartheta^{\star}\right)^{2}}+\frac{1}{4 \vartheta^{\star}}\left(\frac{\varphi^{\prime \prime}(0)}{\varphi^{\prime}(0)}\right)^{2}-\frac{1}{3 \vartheta^{\star}} \frac{\varphi^{\prime \prime \prime}(0)}{\varphi^{\prime}(0)}-\frac{1}{\left(\vartheta^{\star}\right)^{2}} \frac{\varphi^{\prime}(0)}{\psi(\vartheta)}+\frac{1}{\left(\vartheta^{\star}\right)^{3}} \frac{\varphi^{\prime}(0)}{\psi^{\prime}(\vartheta)}\right),
$$

or, more precisely,

$$
\begin{aligned}
\rho(\vartheta) & -\frac{1}{v}\left(-\frac{\varphi^{\prime \prime}(0)}{2\left(\vartheta^{\star}\right)^{2}}+\frac{1}{4 \vartheta^{\star}}\left(\frac{\varphi^{\prime \prime}(0)}{\varphi^{\prime}(0)}\right)^{2}-\frac{1}{3 \vartheta^{\star}} \frac{\varphi^{\prime \prime \prime}(0)}{\varphi^{\prime}(0)}-\frac{1}{\left(\vartheta^{\star}\right)^{2}} \frac{\varphi^{\prime}(0)}{\zeta}\right) \\
& \sim \frac{\sqrt{2} \varphi^{\prime}(0)}{\sqrt{\varphi^{\prime \prime}(\zeta)} v\left(\vartheta^{\star}\right)^{2}}\left(\frac{1}{\zeta^{2}}+\frac{\varphi^{\prime \prime}(\zeta)}{\vartheta^{\star}}\right) \sqrt{\vartheta-\vartheta^{\star}} .
\end{aligned}
$$

We now relate the behavior of a transform $\int_{0}^{\infty} \mathrm{e}^{-\vartheta t} f(t) \mathrm{d} t$ (around a branching point $\vartheta^{\star}<0$ ) to the behavior of the 'transformed' function $f(t)$ (for large $t$ ). We obtain the following result (cf. for instance, the 'Heaviside approach' of [3, Equations (3.21)-(3.23)]); see also [13, pp. 153-154].

Proposition 4.1. Suppose that $\varphi(\alpha)<\infty$ for some $\alpha<0$. Then

$$
r(t) \sim \ell \frac{\mathrm{e}^{\vartheta^{\star} t}}{t \sqrt{t}} \text { as } t \rightarrow \infty
$$


where

$$
\ell:=-\frac{\varphi^{\prime}(0)}{\sqrt{2 \pi \varphi^{\prime \prime}(\zeta)} v\left(\vartheta^{\star}\right)^{2}}\left(\frac{1}{\zeta^{2}}+\frac{\varphi^{\prime \prime}(\zeta)}{\vartheta^{\star}}\right) .
$$

Remark 4.1. As defined in Proposition 4.1, $\ell$ is positive, as is seen as follows. From (3.3) we know that

$$
f(\alpha):=\frac{2}{\varphi^{\prime \prime}(0)} \frac{\alpha \varphi^{\prime}(\alpha)-\varphi(\alpha)}{\alpha^{2}}
$$

is a Laplace transform, and, hence, $-f^{\prime}(\alpha) /-f^{\prime}(0)$ is also; so, for all $\alpha$, it holds that $f^{\prime}(\alpha)<0$, or

$$
\alpha^{3} \varphi^{\prime \prime}(\alpha)-2 \alpha^{2} \varphi^{\prime}(\alpha)+2 \alpha \varphi(\alpha)<0 .
$$

Now insert $\alpha:=\zeta<0$. Using $\varphi^{\prime}(\zeta)=0$ and $\zeta<0$, we obtain $\zeta^{2} \varphi^{\prime \prime}(\zeta)+2 \varphi(\zeta)>0$, which implies that

$$
\frac{\varphi^{\prime \prime}(\zeta)}{\varphi(\zeta)}+\frac{2}{\zeta^{2}}<0
$$

(use $\varphi(\zeta)<0$ ), and, hence,

$$
-\frac{\varphi^{\prime \prime}(\zeta)}{\vartheta^{\star}}>\frac{2}{\zeta^{2}}>\frac{1}{\zeta^{2}}
$$

thus implying that $\ell>0$.

Example 4.1. It can be checked that, for Brownian motion with drift, i.e. $X \in \operatorname{Bm}(-1,1)$ as in the setting of Example 2.1,

$$
r(t) \sim 8 \sqrt{\frac{2}{\pi}} \frac{\mathrm{e}^{-t / 2}}{t \sqrt{t}} ;
$$

this could be found directly from (2.8) as well (cf. [1] and [18, Section 12.1]).

Example 4.2. For the compound Poisson model with exponential jobs (i.e. M/M/1 queue), it can be checked that

$$
\psi(\vartheta)=\frac{1}{2}\left(\lambda-\mu+\vartheta+\sqrt{(\lambda-\mu+\vartheta)^{2}+4 \vartheta \mu}\right),
$$

so that the branching point is $\vartheta^{\star}=-(\sqrt{\mu}-\sqrt{\lambda})^{2}$. Also, $\zeta=-\mu+\sqrt{\lambda \mu}$. Proposition 4.1 now yields an explicit expression for the correlation asymptotics:

$$
r(t) \sim \frac{1}{2 \rho \sqrt{\pi}}\left(\frac{1-\sqrt{\rho}}{1+\sqrt{\rho}}\right)^{3} \frac{\exp [-(1-\sqrt{\rho}) \sqrt{\mu} t]}{(\sqrt{\mu} t)^{3 / 2}} \text { as } t \rightarrow \infty .
$$

Remark 4.2. For compound Poisson input, that is, $X \in \mathrm{CP}(\lambda, \beta(\cdot))$, the tail asymptotics of the correlation function are proportional to those of the busy period, at least in this light-tailed regime (where light tailedness here means that we should require that $\beta(\alpha)<\infty$ for some $\alpha<0$ ). This can be seen as follows.

First recall that the Laplace exponent is $\varphi(\alpha)=\alpha-\lambda+\lambda \beta(\alpha)$. With $\pi(\cdot)$ the Laplace transform of the busy period, it is known that it satisfies $\pi(\vartheta)=\beta(\vartheta+\lambda-\lambda \pi(\vartheta))$. Therefore,

$$
0=\beta(\vartheta+\lambda-\lambda \pi(\vartheta))-\pi(\vartheta)=\frac{1}{\lambda} \varphi(\vartheta+\lambda-\lambda \pi(\vartheta))-\frac{\vartheta}{\lambda},
$$


and, hence, $\varphi(\vartheta+\lambda-\lambda \pi(\vartheta))=\vartheta$. Applying $\psi(\cdot)$ to both sides, we obtain

$$
\pi(\vartheta)=\frac{\lambda+\vartheta}{\lambda}-\frac{1}{\lambda} \psi(\vartheta)
$$

Considering the tail asymptotics of the busy period, first observe that $\pi(\cdot)$ also has a branching point at $\vartheta^{\star}<0$ (i.e. it has the same branching point as $\rho(\vartheta)$ ) such that, for $\vartheta \downarrow \vartheta^{\star}$,

$$
\pi(\vartheta) \sim \frac{\lambda-\vartheta}{\lambda}-\frac{1}{\lambda}\left(\zeta+\sqrt{\frac{2}{\varphi^{\prime \prime}(\zeta)}} \sqrt{\vartheta-\vartheta^{\star}}\right) .
$$

Applying the Heaviside approach now yields, with $P$ the busy period,

$$
\frac{\mathrm{d}}{\mathrm{d} t} \mathrm{P}(P \leq t) \sim \frac{1}{\lambda} \sqrt{\frac{2}{\varphi^{\prime \prime}(\zeta)}} \frac{1}{2 \sqrt{\pi}} \frac{\mathrm{e}^{\vartheta^{\star} t}}{t \sqrt{t}}=\frac{1}{\sqrt{2 \pi}} \sqrt{\frac{1}{\beta^{\prime \prime}(\zeta)}} \frac{\mathrm{e}^{\vartheta^{\star} t}}{\lambda t \sqrt{\lambda t}},
$$

in line with the results of [13, Section 5.6]. These asymptotics are indeed proportional to those of Proposition 4.1. Similarly, applying the relation

$$
\mathrm{E}\left(\mathrm{e}^{-\vartheta P}\right)=1-\vartheta \int_{0}^{\infty} \mathrm{P}(P>t) \mathrm{d} t
$$

we obtain

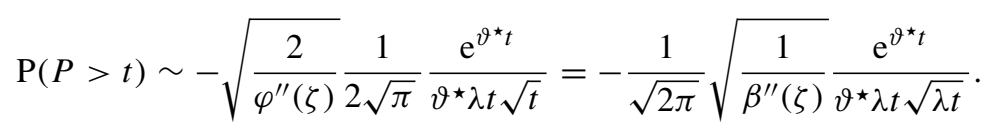

\section{Correlation asymptotics for heavy-tailed input}

Where the previous section focused on light-tailed Lévy input, we now consider the heavytailed case. We extensively use the concept of slowly (and regularly) varying functions. Proposition 5.1, below, is the main result of this section; in Corollary 5.1, below, it is applied to the situation of a queue with $\mathrm{CP}$ input with regularly varying jobs.

The following class of functions plays a crucial role in our analysis.

Definition 5.1. We say that $f(x) \in \mathcal{R}_{\delta}(n, \sigma)$, where $n \in \mathbb{N}, \sigma \in \mathbb{R}$, and $\delta \in(n, n+1)$, for $x \downarrow 0$ if

$$
f(x)=\sum_{i=0}^{n} \frac{f^{(i)}(0)}{i !} x^{i}+\sigma x^{\delta} L\left(\frac{1}{x}\right) \quad(x \downarrow 0)
$$

for a slowly varying function $L$ (i.e. $L(x) / L(t x) \rightarrow 1$ for $x \rightarrow \infty$ and any $t>0$ ).

Lemma 5.1. Suppose that $\varphi^{\prime}(\alpha) \in \mathcal{R}_{\delta-1}(n-1, \sigma)$. Then, for $\alpha \downarrow 0$,

$$
\varphi(\alpha) \in \mathcal{R}_{\delta}\left(n, \frac{\sigma}{\delta}\right)
$$

and, for $\vartheta \downarrow 0$,

$$
\begin{gathered}
\psi(\vartheta) \in \mathcal{R}_{\delta}(n, \tau) \text { with } \tau:=-\frac{\sigma}{\delta\left(\varphi^{\prime}(0)\right)^{\delta+1}}, \\
\psi^{\prime}(\vartheta) \in \mathcal{R}_{\delta-1}(n-1, \tau \delta)
\end{gathered}
$$


Proof. The first statement is an immediate consequence of Karamata's theorem; the second statement follows from $\psi(\varphi(\alpha))=\alpha$; the third statement follows in an elementary way by using $\psi^{\prime}(\vartheta)=1 / \varphi^{\prime}(\psi(\vartheta))$.

The following lemma presents the behavior of $\xi_{\mathrm{e}}(\vartheta)$ as $\vartheta \downarrow 0$. We need this type of result as Karamata's Tauberian theorem then enables us to translate the behavior of transforms around 0 into the behavior of $r(t)$ for large $t$.

Lemma 5.2. If $\varphi^{\prime}(\alpha) \in \mathcal{R}_{\delta-1}(n-1, \sigma)$, with $n \in\{3,4, \ldots\}$ and $\delta \in(n, n+1)$, then

$$
\xi_{\mathrm{e}}(\vartheta)=1-\vartheta \rho(\vartheta) \in \mathcal{R}_{\delta-3}(n-3, \omega) \quad \text { with } \quad \omega:=\frac{(\delta-1)}{v \delta\left(\varphi^{\prime}(0)\right)^{\delta-2}} \sigma .
$$

Proof. Recall (3.1) and (3.2). The crucial step is to verify that

$$
\frac{\vartheta}{\psi(\vartheta)} \in \mathcal{R}_{\delta-1}\left(n-1,-\tau\left(\varphi^{\prime}(0)\right)^{2}\right) \quad \text { and } \quad \frac{1}{\psi^{\prime}(\vartheta)} \in \mathcal{R}_{\delta-1}\left(n-1,-\tau \delta\left(\varphi^{\prime}(0)\right)^{2}\right) ;
$$

use Lemma 5.1. Verification of the claim is now straightforward (though tedious).

The Tauberian theorem in [12, Theorem 8.1.6] now yields the following result; see also [10].

Proposition 5.1. If $\varphi^{\prime}(\alpha) \in \mathcal{R}_{\delta-1}(n-1, \sigma)$, with $n \in\{3,4, \ldots\}$ and $\delta \in(n, n+1)$, then

$$
r(t) \sim \frac{\omega(-1)^{n+1}}{\Gamma(4-\delta)} t^{3-\delta} L(t) \quad \text { as } t \rightarrow \infty .
$$

Proof. First recall that $r(t)=\mathrm{P}\left(Z_{\mathrm{e}}>t\right)$ and that $Z_{\mathrm{e}}$ has transform $\xi_{\mathrm{e}}(\cdot)$. Lemma 5.2 and Theorem 8.1.6 of [12] yield the result.

Corollary 5.1. Interestingly, we can now also find a criterion for long-range dependence; $c f$. the remarks in the introduction of [22].

Suppose that $\varphi^{\prime}(\alpha) \in \mathcal{R}_{\delta-1}(n-1, \sigma)$, with $n \in\{3,4, \ldots\}$ and $\delta \in(n, n+1)$. Then the queueing process is long-range dependent if $n=3$, as in this case $\int_{0}^{\infty} r(t) \mathrm{d} t=\infty$. Consider, for instance, the case in which $X \in \mathrm{CP}(\lambda, \beta(\cdot))$, with $\mathrm{P}(B>t) \sim t^{-v}$ for $v \in(3,4)$. Then the first three moments of $B$ exist and, hence, also the first two moments of the steady-state queue length, as well as the covariance $\operatorname{cov}\left(Q_{0}, Q_{t}\right)$. The tail of $B$, however, is so heavy that $\operatorname{cov}\left(Q_{0}, Q_{t}\right)$ decays roughly as $t^{3-v}$, giving rise to a long-range dependent queueing process.

Likewise, it follows that the queueing process is short-range dependent if $n \in\{4,5, \ldots\}$; for instance, when considering $C P$ input with $\mathrm{P}(B>t) \sim t^{-v}$ for $v \in(4, \infty)$.

\section{Concluding remarks}

In this paper we studied the correlation function of the workload process of a single queue fed by a Lévy process (that is, a Lévy process reflected at 0 ). Relying on the concept of complete monotonicity we have been able to derive a set of structural properties of the correlation function, viz. that it is a positive, decreasing, and convex function. Importantly, we have shown how to represent the correlation function $r(\cdot)$ as the complementary distribution function of a specific random variable. This representation, as well as an explicit characterization of the Laplace transform of $r(\cdot)$, enabled the analysis of the asymptotic behavior of $r(t)$ for large $t$; both the light-tailed and heavy-tailed cases were studied.

An alternative way to conclude that the correlation function is positive, decreasing, and convex, may be the following. The Laplace exponent of any Levy process can be approximated 
arbitrarily closely by that of an appropriately chosen CP process; see, e.g. [14, Theorem XVII.1]. As the claim has been proved for CP input [22], a limit argument may lead to an alternative proof of Theorem 3.1. Exploration of this approach is a subject for further research.

Restricting ourselves to the case of CP input, we could say that Section 4 covers the case in which the jobs have a finite moment generating function in a neighborhood of the origin: $\beta(\alpha)<\infty$ for some $\alpha<0$, and, hence, all moments are finite. On the other hand, in Section 5 we addressed the situation in which just a finite set of moments are finite. In between, however, there is a third class of distributions: those for which all moments are finite, but without an analytic continuation for $\alpha<0$ (that is, $\beta(\alpha)=\infty$ for all $\alpha<0$ ). Examples of distributions in this class are the Weibull and lognormal distributions. A subject for further research would be the analysis of the correlation asymptotics for this class of distributions.

As we lack, in most cases, an explicit formula for $r(t)$, we may attempt to estimate it through simulation. This is particularly challenging, as $r(t)$ can be extremely small for large $t$, and is the difference of two (potentially large) numbers. A way to circumvent this problem is to use importance sampling [5, Section V.1], that is, sampling under an alternative measure and correcting the simulation output by likelihood ratios (that keep track of the relative likelihood of the realization under the actual measure, relative to the alternative measure). The resemblance with the busy period asymptotics suggests that, for light-tailed input, the (exponentially-twisted) change of measure proposed in [20] may work well; it is noted that the analysis of [20] indicates that the twisting of the work present at time 0 should be handled with care. Another option could be to rely on the representation of the correlation function $r(\cdot)$ as the complementary distribution function of the random variable $Z_{\mathrm{e}}$; see Theorem 3.1.

A potential application area of our results is the following. Suppose that no measurements of the queue's input process can be made, and, hence, estimation of the probabilistic law of the input process has to be performed in an alternative manner. One approach could be to measure the queue's workload (for instance periodically), and to infer the input characteristics from the resulting measurements. Insight into the correlation between subsequent measurements, as obtained in the present paper, may be useful when devising such a procedure. Work along these lines for queues with Gaussian input was carried out by Mandjes and van de Meent [19] (in a somewhat more experimental context), and for M/G/ $\infty$ systems by Bingham and Pitts [11] (building on the results presented in [23]); see also [15].

\section{Appendix A. Complete monotonicity}

The concept of complete monotonicity is summarized in the following definition.

Definition A.1. A function $f(\alpha)$ on $[0, \infty)$ is completely monotone if, for all $n \in \mathbb{N}$ and $\alpha \geq 0$,

$$
(-1)^{n} \frac{\mathrm{d}^{n}}{\mathrm{~d} \alpha^{n}} f(\alpha) \geq 0 .
$$

We write $f(\alpha) \in \mathcal{C}$.

The following deep and powerful result is due to Bernstein [7]. It says that there is equivalence between $f(\alpha)$ being completely monotone and the possibility of writing $f(\alpha)$ as a Laplace transform. For more background on completely monotone functions, see [14, pp. 439-442].

Theorem A.1. A function $f(\alpha)$ on $[0, \infty)$ is the Laplace transform of a nonnegative random variable if and only if (i) $f(\alpha) \in \mathcal{C}$ and (ii) $f(0)=1$. 
The concept of complete monotonicity is easy to work with, as we can use a set of practical properties.

Lemma A.1. The following properties apply.

(i) $\mathcal{C}$ is closed under addition: if $f(\alpha) \in \mathcal{C}$ and $g(\alpha) \in \mathcal{C}$ then $f(\alpha)+g(\alpha) \in \mathcal{C}$. This extends to, if $f_{x}(\alpha) \in \mathcal{C}$ for $x \in \Xi, \int_{x \in \Xi} f_{x}(\alpha) \mu(\mathrm{d} x) \in \mathcal{C}$ for any measure $\mu(\cdot)$.

(ii) $\mathcal{C}$ is closed under multiplication: if $f(\alpha) \in \mathcal{C}$ and $g(\alpha) \in \mathcal{C}$ then $f(\alpha) g(\alpha) \in \mathcal{C}$.

(iii) Properties of composite $\mathcal{C}$ functions: if $f(\alpha) \in \mathcal{C}$ and $g(\alpha) \geq 0$ with $g^{\prime}(\alpha) \in \mathcal{C}$, then $f(g(\alpha)) \in \mathcal{C}$.

(iv) Let $U(\alpha)$ be nondecreasing on $[0, \infty)$, let $U(0)=0$, let $u:=\lim _{\alpha \rightarrow \infty} U(\alpha)<\infty$, and let

$$
f(\alpha):=\int_{[0, \infty)} \mathrm{e}^{-\alpha x} \mathrm{~d} U(x) ;
$$

clearly, $f(\alpha) \in \mathcal{C}$ and $u=f(0)$. Then also

$$
g(\alpha):=\frac{f(0)-f(\alpha)}{\alpha} \in \mathcal{C} .
$$

(v) $\mathcal{C}$ is closed under differentiation: if $f(\alpha) \in \mathcal{C}$ then $-f^{\prime}(\alpha) \in \mathcal{C}$.

Proof. Property (i) follows trivially from the definition. Property (ii) follows from [14, Criterion 1], and property (iii) follows from [14, Criterion 2]. Property (iv) can be found in, for instance, [22, Equation (4.2)]. The proof of property (v) is trivial.

Lemma A.2. Let $\left(Y_{t}\right)_{t \geq 0}$ be an increasing subordinator Lévy process, with Laplace exponent $\xi(\alpha)$. Then $-\xi^{\prime}(\alpha) \in \mathcal{C}$.

Proof. According to Bertoin [8, Chapter III, Equation (3)], we can write

$$
\xi(\alpha)=-d \alpha+\int_{(0, \infty)}\left(\mathrm{e}^{-\alpha x}-1\right) \Pi_{\xi}(\mathrm{d} x),
$$

with $d \geq 0$ and measure $\Pi_{\xi}(\cdot)$ such that $\int_{(0, \infty)} \min \left\{1, x^{2}\right\} \Pi_{\xi}(\mathrm{d} x)<\infty$. This implies that

$$
-\xi^{\prime}(\alpha)=d+\int_{(0, \infty)} x \mathrm{e}^{-\alpha x} \Pi_{\xi}(\mathrm{d} x),
$$

so that $-\xi^{\prime}(\alpha) \in \mathcal{C}$; use Lemma A.1(i).

\section{Acknowledgements}

The authors thank Peter Glynn (Stanford University) and Teun Ott (Winlab, Rutgers University) for valuable remarks.

\section{References}

[1] Abate, J. And Whitt, W. (1988). The correlation functions of RBM and M/M/1. Stoch. Models 4, 315-359.

[2] Abate, J. And Whit, W. (1994). Transient behavior of the M/G/1 workload process. Operat. Res. 42, 750-764.

[3] Aвate, J. And Whitt, W. (1997). Asymptotics for M/G/1 low-priority waiting-time tail probabilities. Queueing Systems 25, 173-233. 
[4] Asmussen, S. (2003). Applied Probability and Queues, 2nd edn. Springer, New York.

[5] Asmussen, S. And Glynn, P. (2007). Stochastic Simulation: Algorithms and Analysis (Stoch. Modelling Appl. Prob. 57). Springer, New York.

[6] Beneš, V. (1957). On queues with Poisson arrivals. Ann. Math. Statist. 28, 670-677.

[7] Bernstein, S. N. (1929). Sur les fonctions absolument monotones. Acta Math. 52, 1-66.

[8] Bertoin, J. (1996). Lévy Processes (Camb. Tracts Math. 121). Cambridge University Press.

[9] Bingham, N. H. (1975). Fluctuation theory in continuous time. Adv. Appl. Prob. 7, 705-766.

[10] Bingham, N. H. And Doney, R. A. (1974). Asymptotic properties of subcritical branching processes. I. The Galton-Watson process. Adv. Appl. Prob. 6, 711-731.

[11] Bingham, N. H. and Pitts, S. M. (1999). Non-parametric estimation for the M/G/ $\infty$ queue. Ann. Inst. Statist. Math. 51, 71-97.

[12] Bingham, N. H., Goldie, C. M. and Teugels, J. L. (1987). Regular Variation (Encyclopaedia Math. Appl. 27). Cambridge University Press.

[13] Cox, D. R. AND Smith, W. L. (1961). Queues. Methuen, London.

[14] Feller, W. (1971). An Introduction to Probability Theory and Its Applications, Vol. 2, 2nd edn. John Wiley, New York.

[15] Hall, P. G. and Park, J. (2004). Nonparametric inference about service time distributions from indirect measurements. J. R. Statist. Soc. B66, 861-875.

[16] Kella, O., Boxma, O. J. And Mandjes, M. (2006). A Lévy process reflected at a Poisson age process. J. Appl. Prob. 43, 221-230.

[17] Kyprianou, A. (2006). Introductory Lectures on Fluctuations of Lévy Processes with Applications. Springer, Berlin.

[18] MandJEs, M. (2007). Large Deviations for Gaussian Queues. John Wiley, Chichester.

[19] Mandjes, M. and van de Meent, R. (2008). Resource dimensioning through buffer sampling. To appear in IEEE/ACM Trans. Networking.

[20] MandJEs, M. ANd Zwart, B. (2006). Large deviations for sojourn times in processor sharing queues. Queueing Systems 52, 237-250.

[21] Morse, P. (1955). Stochastic properties of waiting lines. Operat. Res. 3, 255-261.

[22] Отт, T. (1977). The covariance function of the virtual waiting-time process in an M/G/1 queue. Adv. Appl. Prob. 9, 158-168.

[23] Reynolds, J. F. (1975). The covariance structure of queues and related processes-a survey of recent work. Adv. Appl. Prob. 7, 383-415.

[24] SAto, K. (1999). Lévy Processes and Infinitely Divisible Distributions (Camb. Studies Adv. Math. 68). Cambridge University Press.

[25] Zolotarev, V. (1964). The first passage time of a level and the behaviour at infinity for a class of processes with independent increments. Theory Prob. Appl. 9, 653-661. 